\title{
Envejecimiento, género y cuidados: debates para situar las políticas públicas
}

\section{Envelhecimento, gênero e cuidados: debates para situar as políticas públicas}

\section{Aging, Gender and Care: Debates to Situate Public Policies}

(iD) Herminia Gonzálvez Torralbo

Universidad Central de Chile, Santiago de Chile, Chile.

herminiagonzalvez@gmail.com

iD Sofia Larrazabal

Centro de Justicia Educacional, Santiago de Chile, Chile.

sofia.larrazabal@gmail.com

iD (9) Menara Guizardi

Consejo Nacional de Investigaciones Científicas y Tecnológicas, Buenos Aires, Argentina y Universidad de Tarapacá, Arica, Chile.

menaraguizardi@yahoo.com.br

Resumen: Presentamos un estado del arte que sitúa los debates sobre cuidados en países que atraviesan procesos demográficos de envejecimiento. Su objetivo es aportar, desde una perspectiva transversal de género, a la formulación de políticas públicas que hagan frente a los desafíos planteados por la transición demográfica en Chile. Partiremos por revisar los debates sobre el envejecimiento, sus dimensiones de género y el concepto de cuidados en las ciencias sociales. Revisaremos, además, los estudios sobre las necesidades de cuidado de las personas mayores, analizando el rol de la familia, del Estado, de la comunidad y del mercado en la atención de estas demandas sociales. Finalizamos 
discutiendo algunos puntos críticos a ser considerados para la planificación de políticas públicas vinculadas al tema.

Palabras clave: Envejecimiento. Género. Cuidados. Política pública. Chile.

Resumo: Apresentamos uma revisão de estado da arte que situa os debates sobre cuidados nos países que atravessam processos demográficos de envelhecimento. O objetivo é aportar, a partir de uma perspectiva transversal de gênero, à formulação de políticas públicas que deem solução aos desafios da transição de demográfica em países como Chile. Revisaremos os debates sobre envelhecimento, suas dimensões de gênero e o conceito de cuidados nas ciências sociais. Revisaremos, ademais, os estudos sobre as necessidades de cuidado das pessoas idosas, analisando o papel da família, Estado, comunidade e mercado na atenção destas demandas sociais. Finalizaremos discutindo pontos críticos a ser considerados para a planificação de políticas públicas vinculadas ao tema.

Palavras-chave: Envelhecimento. Gênero. Cuidados sociais. Políticas públicas. Chile.

Abstract: The article presents a state of the art that situates the relationship between the processes of population aging and the demands of care. Its objective is to contribute, from a transversal gender perspective, to the formulation of public policies that face the challenges posed by the demographic transition in Chile. We will start by reviewing the debates on aging, its gender dimensions, and the concept of care. We will also review the studies on the care needs of the elderly, analyzing the role of the family, the State, the community, and the market in addressing these social demands. We conclude discussing a few critical points to be considered for the planning of public policies linked to the topic.

Keywords: Aging. Gender. Social care. Public policies. Chile.

Data de recebimento: 04/08/2018

Data de aprovação: 28/04/2020 


\section{Introducción}

El siglo XXI constituye una era de "implosión geriátrica" y de "globalización del envejecimiento" (AGAR, 2001, p.31). Estos fenómenos se vinculan estrechamente con la reducción de las tasas de natalidad y mortalidad (CEPAL, 2000), con el aumento de la esperanza de vida, y con la consecuente distensión de los ciclos vitales de la población mundial (ARANCO et al., 2018, p.7). Parte de estas tendencias se confirman en América Latina. En el 2000, 8,1\% de la población regional tenía 60 años o más, mientras, en 2015, este índice ya alcanzaba el 11,1\%. Se proyecta que la cifra llegue a 25,4\% en 2050 (BCN, 2016). No obstante, los países latinoamericanos experimentan el envejecimiento de manera heterogénea. La causa debe buscarse en las diversas formas de abordar -desde las políticas públicas o estatales- las transformaciones sociales que devienen de la reducción de las tasas de natalidad y mortalidad y el aumento de la esperanza de vida. Sin embargo existe una pluralidad de factores que impactan su composición demográfica: la organización territorial de sus actividades económicas, el rol económico que desempeñan a nivel regional, sus patrones migratorios internacionales y sus dinámicas migratorias intranacionales.

Atentos a estas especificidades, diversos estudios vienen abordando los efectos del envejecimiento latinoamericano indagando sobre procesos locales y nacionales (GANGA et al., 2016; GONZÁLVEZ, 2017; RODRíGUEZ et al., 2017). El caso de Chile se viene presentando como paradigmático. Para el año 2000, el país atravesaba "una fase de transición demográfica avanzada reciente" (MARCO, 2004, p.49): su pirámide poblacional tenía una base más estrecha que el cuerpo medio; la población infantil y adolescente era menos voluminosa que la joven y adulta (efecto del descenso en las tasas de fecundidad entre 1980 y 2000). Actualmente, Chile se posiciona a la par de países de la región como Argentina, Uruguay, Cuba y Brasil, que atraviesan un estado avanzado de transición 
demográfica (GANGA et al., 2016, p.197), caracterizado por cinco indicadores:

- Un elevado índice de esperanza de vida de la población al nacer, superior a los 80 años. Para el quinquenio 2005-2010, Chile presentaba la mayor tasa de América Latina: 79,4 años. En 2016, alcanzó los 80,5 años, solo siendo superado en el continente americano por Canadá (OMS, 2016). Las estimaciones proyectan que Chile se mantendrá como el país latinoamericano con mayor potencial de longevidad para el periodo 2045-2050, llegando a una esperanza de vida de 87,9 años (CELADE, 2014).

- Elevadas proporciones del Índice de Adulto Mayor (IAM). EI IAM establece la proporción de personas mayores por cada 100 habitantes menores de 15 años. Para el año 1990, el IAM apuntaba la existencia de 30 personas mayores por cada 100 niños en Chile. En 2010, la proporción pasó a 59,1/100 y, en 2012, a 67,1/100 (GANGA et al., 2016, p.182).

- Ascenso de la proporción de población mayor con relación a la población absoluta derivadas del último Censo (2017). De un total de 17.574.003 personas computadas en el país, 2.850.171 tenían 60 años o más (INE, 2017).

- Constatación del incremento de las desigualdades sociales entre los y las chilenas en la vejez. En el diagnóstico sobre la desigualdad en Chile desarrollado por el Programa de las Naciones Unidas para el Desarrollo (PNUD, 2017), el estudio apunta que cerca del $72 \%$ de los jubilados en el país recibe una pensión inferior al valor mínimo (definido como el $70 \%$ del salario mínimo nacional) (PNUD, 2017, p.25). Las bajas pensiones generan una sensación de inseguridad ciudadana, especialmente para las poblaciones de los dos quintiles más pobres. Menos de la mitad de los encuestados de esta población cree que contará con los recursos suficientes en su vejez. La jubilación de estos mayores representa, en promedio, el $60 \%$ de los ingresos de su hogar, lo que indica que se hacen cargo económicamente de sus familias (PNUD, 2017, p.333). El descontento generalizado por las desigualdades del sistema 
jubilatorio vigente en Chile tuvo un papel central en las recientes revueltas ciudadanas que se tomaron las calles de todo el país en 2019.

- El incremento de las desigualdades de género entre las poblaciones envejecidas. Según el Censo 2017, hay en Chile 95,9 hombres por cada 100 mujeres. Pero, la proporción femenina se incrementa en la población más envejecida. Entre los mayores de 60 años, 56,8\% son mujeres y 43,13\% son hombres (INE, 2017). Las mujeres de esta franja etaria están menos cubiertas por la jubilación (PNUD, 2017, p.25) y, cuando lo están, reciben valores un 30\% inferiores a los masculinos (PNUD, 2017, p.333).

Juntos, estos indicadores muestran que: i) los y las chilenas viven cada vez más; ii) la población mayor se incrementa dinámicamente y de forma rápida con relación a la joven; iii) y constituye parte significativa de la composición demográfica del país; iv) Que la tercera edad viene siendo empujada a una situación de empobrecimiento y discriminación: el envejecimiento es un factor agravante de las desigualdades económicas y sociales; v) La situación de las mujeres mayores es aún más preocupante, pues las brechas de género se magnifican en la vejez. La inversión de la pirámide poblacional chilena (CHACKIEL, 2000; MARCO, 2004; DONOSO et al., 2009) configura una "crisis demográfica" caracterizada por la "feminización del envejecimiento" (KEHL y FERNÁNDEZ, 2001, p.132).

Estas transformaciones afectan los modos concretos de actuar de toda la sociedad con relación a la economía, a la política y a las configuraciones culturales (morales, axiológicas, simbólicas, discursivas y de prácticas sociales) (KEHL y FERNÁNDEZ, 2001, 133). Se observa, así, una reorganización de las relaciones de dependencia y cuidados que serán asumidas diferenciadamente por los diversos grupos etarios que componen la población chilena. El carácter intergeneracional y transversal de los impactos del envejecimiento demanda planificaciones y respuestas estatales que articulen la participación de diferentes actores sociales. La situación convoca tanto al Estado como a la sociedad civil a la 
Envejecimiento, género y cuidados: debates para situar las políticas públicas Herminia Gonzálvez Torralbo • Sofia Larrazabal • Menara Guizardi

responsabilidad de construir políticas públicas que avancen en la búsqueda de soluciones -de corto, mediano y largo plazo- a la crisis de los cuidados sociales.

Cuando nos referimos a las políticas públicas, aludimos a la definición que las vincula a la intensificación de los mecanismos de participación en los Estados Democráticos de Derecho (VELÁSQUEZ-GAVILANES, 2009, p.151). El término refiere al conjunto de decisiones, actuaciones, directrices y proyecciones estratégicas -explícitas, permanentes, sistemáticas e integradas- legalizados e institucionalizados por el Estado. Lo que distingue las políticas públicas de las demás formas de actuación estatal es que ellas deben ser consensuadas socialmente y surgen para dar respuesta a un problema o contexto social específico, subsanando demandas sociales. Así, aunque deben ser institucionalizadas o legitimadas por el Estado, ellas pueden ser ejecutadas (su diagnóstico, diseño, implementación, y evaluación) por toda una pluralidad de actores: 1) Organismos/organizaciones/actores estatales; 2) Actores de la sociedad civil (asociaciones populares, sindicatos, cooperativas); 3 ) Grupos estudiantiles; 4) Entidades del tercer sector (ONG, ONGD, fundaciones) y 5) organizaciones religiosas.

Este artículo deviene de una investigación de estado del arte ${ }^{1}$ y tiene como objetivo contribuir a la construcción de las políticas públicas sobre el envejecimiento en Chile, ofreciendo una síntesis de debates teóricos que sitúan la relación entre los procesos de envejecimiento poblacional, la sobrecarga de género y las demandas de cuidados que ambos suscitan. Comprendemos que esta síntesis constituye un insumo susceptible de ser usado de manera transversal por los actores convocados a constituir estas nuevas políticas del envejecimiento en el país; con el potencial de aportar a la pluralización de las referencias que amparan estos debates.

\footnotetext{
1 Hemos analizado cerca de 100 textos académicos -entre artículos, capítulos, libros, informes y tesis- en el marco de nuestra revisión de estado del arte. (En el presente texto, citamos directamente a 72 de estas obras). Nuestra selección de este material estuvo orientada por dos criterios centrales. El primero de ellos, temático: buscamos aquellas publicaciones que abordaran las políticas públicas sobre el envejecimiento desde una perspectiva de género. El segundo, de carácter geográfico: concentramos nuestros esfuerzos en la literatura producida en América Latina y enfocamos la lectura en los textos referentes al contexto chileno. Asimismo, realizamos una revisión de la producción anglófona sobre estos temas, buscando con esto poder establecer comparaciones entre las perspectivas analíticas desarrolladas en diferentes contextos globales.
} 
Envejecimiento, género y cuidados: debates para situar las políticas públicas Herminia Gonzálvez Torralbo • Sofia Larrazabal • Menara Guizardi

Presentaremos, en el segundo apartado (subsecuente a esta introducción), una discusión de las investigaciones sobre envejecimiento realizadas en Latinoamérica y en países del Norte global. A través de una perspectiva transversal de género², correlacionaremos estas reflexiones con las casuísticas chilenas, señalando los significados sociales y políticos del envejecimiento que deben ser tomados en consideración para el tratamiento del tema en el país. Analizaremos el concepto de cuidado, especificando las particularidades que este adquiere en contextos de feminización del envejecimiento. El tercer apartado discute las necesidades de las personas mayores, describiendo la "arquitectura a través de la cual se provee el cuidado" en Chile (RODRÍGUEZ, 2015, p.40), configurada por cuatro actores: familia, Estado, comunidad y mercado (GONZÁLVEZ, 2017). Finalizamos señalando puntos críticos a ser considerados para la planificación de políticas públicas.

\section{Vejez, género y cuidados}

Desde 1960, la vejez constituyó un tema marginado en la investigación sociológica, antropológica y politológica (CALASANTI et al., 2006, 13). Aunque los primeros estudios preocupados por las dimensiones sociales del fenómeno son de inicios del siglo XX -y devienen de la medicina, no de las ciencias sociales (RAMOSTORO, 2015, p.71)- el envejecimiento aún representa un campo de conocimiento periférico (MARSHALL, 2006, p.7).

Las definiciones del concepto de vejez suscitaron disensos importantes. El criterio más recurrente para determinar quién se encuentra o no en esta etapa viene siendo la edad de jubilación. Se trata, por lo tanto, de un indicador establecido con base a percepciones sociales y políticas sobre las necesidades productivas de los países en determinado momento. Indicadores vinculados

2 Discutiremos el concepto de género con más detalle en los apartados siguientes, por ahora, solo adelantaremos que la categoría alude a la construcción cultural de las diferencias y desigualdades entre los sexos. 
a otros criterios -la salud o capacidad física de la población, por ejemplo-podrían alterar sustancialmente una definición de la edad jubilatoria establecida desde el punto de vista productivo (LEVY, 1988; KEHL y FERNÁNDEZ, 2001). Como todo fenómeno social, el envejecimiento es dinámico, tiene consecuencias económicas y políticas en lo que concierne a las investigaciones y a la formulación de políticas públicas: su definición debe plantearse de forma historizada y contextual (KNODEL y OFTEDAL, 2003, pp.693-694).

Pese al disenso y a la heterogeneidad de los debates, en las investigaciones realizadas en Europa y las Américas predominan definiciones pesimistas del envejecimiento que lo asocian a la dependencia (HUTHEESING, 1993, p.98), al fracaso personal, a la pérdida de atractivo físico y a la pobreza (ROSENTHAL, 1990; CALASANTI et al., 2006). Los estudios tienden a replicar -frecuentemente de forma acrítica- ciertos imaginarios predominantes en los contextos donde fueron producidos (HUTHEESING, 1993; KEHL y FERNÁNDEZ, 2001). Predominan aquellos estudios que consideran que quienes envejecen pierden paulatinamente aquellos roles y atributos que antes los hacían visibles o prominentes como miembros de la comunidad. La vejez es frecuentemente representada, en las ciencias sociales, como una pérdida de la "razón de ser" de los sujetos (LEVY, 1988, pp.480-481), asumiéndose que la infancia y juventud son las fases determinantes de la vida.

Desde fines de los sesenta, diversos investigadores empezaron a problematizar estas lecturas, alertando que ellas se basan en imaginarios de discriminación por edad. BUTLER (1969) acuñó el término "Age-Ism" (edadismo) para referirse a estos imaginarios, observando que ellos redundan, -además de en la poca atención dada a la vejez en las ciencias-, en la invisibilización del papel de las personas mayores en los diversos ámbitos sociales. El "edadismo" indujo a que muchos investigadores tendieran a generalizar las necesidades y demandas de la tercera edad sin considerarlas interseccionalmente a la luz de categorías como género, clase y raza (CALASANTI et al., 2006, p.25). 
En Chile, diversos autores concuerdan con que las definiciones de la vejez en las ciencias sociales están tácitamente influidas por imaginarios sociales que la encasillan como una etapa improductiva y frágil -de dependencia, pasividad, declive sexual y dificultades de adaptación a nuevos contextos sociales y tecnológicoscontrastando, así, con representaciones sobre la "ventaja" de la juventud. Estos estereotipos impactan la experiencia vital de los mayores. Estudios comprueban, por ejemplo, que afectan su inserción al mercado laboral chileno, dada la generalización de percepciones que vinculan el envejecimiento con un menor potencial de desarrollo laboral, creatividad o motivación; con lentitud en la toma de decisiones y deterioro físico (NAZAR y FIGUEROA, 2015, p.124).

No obstante, todavía se carece de un análisis, desde las ciencias sociales, que articule los impactos y debilidades de las políticas públicas de vejez en el país desde una perspectiva crítica al edadismo. En el Norte global, estudios de este tipo empezaron a producirse ya en fines de los setenta. El trabajo de ESTES (1979) fue pionero en este campo, concluyendo que en esta relación influyen: 1) las percepciones, los mitos y los mensajes sobre el envejecimiento comunicados tanto a viejos como a jóvenes por los medios y los líderes de opinión, incluyendo a los investigadores (ESTES, 1979, p.290); 2) El modo general en que la sociedad trata a sus mayores a través de las políticas sociales de empleo, jubilación, cuidado de salud, ingresos y familia (ESTES, 1979, p.292). Así, las políticas sociales aplicadas a la vejez estarían impactadas tanto por imaginarios sociales que la representan como un "problema", como por discursos y representaciones que la retratan como una "contrariedad" médica a ser aliviada (o erradicada) a través de medicaciones (ESTES et al., 1984). ESTES (1993, p.292) observó, además, que las construcciones sociales sobre el envejecimiento desde las políticas sociales reflejan y reproducen disparidades de clase social, género, raza y etnicidad (HESS, 1985; PEACE, 1986; COLEMAN Y WATSON, 1987). 
Envejecimiento, género y cuidados: debates para situar las políticas públicas Herminia Gonzálvez Torralbo • Sofia Larrazabal • Menara Guizardi

Pero hay una dimensión de género vinculada al "Age-Ism" que es particularmente importante en lo que concierne a la producción de políticas públicas. La introducción de una perspectiva de género sobre estas cuestiones fue tardía (MARSHALL, 2006, p.8), implicando la reproducción de lecturas androcéntricas (GIBSON, 1996, p.433). Con "perspectiva de género", aludimos a la orientación analítica en las ciencias sociales que define al género como la construcción cultural de la diferencia biológica entre lo masculino y lo femenino, estructurada a través de un campo conflictivo: activando procesos de dominio que repercuten sobre mujeres y hombres, generando disputas simbólicas que configuran las diferencias, inclusiones y exclusiones (LAMAS, 1999, p.147).

Los estudios del envejecimiento desarrollados desde la gerontología contemplaban, desde 1940, algunas reflexiones sobre las diferencias entre las experiencias masculinas y femeninas (OSORIO, 2006, p.7). Con todo, es solo casi cinco décadas más tarde que observamos un giro epistémico que centraliza la dimensión femenina del fenómeno en las investigaciones sociales (OSORIO, 2006, p.6) ${ }^{3}$. Este giro se refiere al análisis de las intersecciones entre género y envejecimiento: comienzan con la suposición de que el género y la edad constituyen, ambos, construcciones sociales que inciden directamente en el curso de vida (LEVY, 1988, pp.484485). Son, además, categorizaciones poderosas y penetrantes en la estructuración de las interacciones humanas: "forman la base sobre la cual las identidades de las personas se establecen y sobre la cual comienzan las líneas de comunicación y locación social" (LEVY, 1988, p.480). Los significados negativos asociados a las personas mayores afectan a las mujeres diferencialmente, debido a su condición de doble subalternidad: de género y de edad.

Con todo, abundan las investigaciones sociales que representan a la vejez como "un tiempo en que las agudas distinciones de sexo presentes en la infancia, la adolescencia y la adultez temprana menguan" (DATAN, 1989, p.15). Este tipo de lecturas deben ser

3 Las aportaciones feministas sobre la vejez tardaron en aparecer (Rosenthal, 1990). Cuando lo hicieron, siguieron ocupando un lugar marginal entre las pautas de investigación, reproduciendo el imaginario de la vejez femenina como "problema social" (Datan, 1989; Marshall, 2006). 
problematizadas en la construcción de políticas públicas, las cuales debieran poner atención en las particularidades del envejecimiento femenino. Los estudios previos sobre el tema permiten establecer algunos contornos generales del fenómeno. Por ejemplo, las mujeres viven, en promedio, más que los hombres. En las relaciones heterosexuales, tienden a casarse con compañeros mayores que ellas, lo que repercute en la distención de su periodo de viudez (DATAN, 1989, p.16). Tienden también a ser quienes cuidan de sus maridos cuando estos envejecen (ZARIT et al., 1998, pp.499-500). La mortalidad de un hombre aumenta considerablemente en el primer año de viudez, lo que no se verifica entre las mujeres: ellas son más eficientes en la mantención de las redes sociales que sustentan la vida (KNODEL y OFSTEDAL, 2003, p.680).

Estas cuestiones permiten indagar sobre la relación entre cuidados y envejecimiento en contextos en los que ambos se presentan feminizados-como en Chile-. Los "cuidados" constituyen una categoría polisémica, vinculable a diversas formas de acción (DUFFY, 2011, p.9). Equivalen a "las actividades y relaciones que se desarrollan con el fin de satisfacer las necesidades físicas y emocionales de las personas" (DEL VALLE, 2013, p.30). Son multidimensionales y cotidianos, engendrando todos los aspectos necesarios para la sostenibilidad (social y orgánica) de la vida (HUENCHUÁN, 2014, p.153). Así, las necesidades de cuidado son básicas para la reproducción de la vida y tienen un carácter intergeneracional (GLENN, 2010, p.5). Miembros de diferentes generaciones se cuidan entre sí, porque la necesidad de ser cuidado atraviesa todos los ciclos vitales, aunque transformándose (IZQUIERDO, 2003, 12). El cumplimiento de estas necesidades puede hacerse de forma continuada o esporádica, pero apunta, frecuentemente, a resolver situaciones de dependencia (COMAS, 2017, p.20). El acto de cuidar y de ser cuidado involucra, además, la producción de afectos (ENGLAND, 2005, p.389) y de responsabilidades combinadas (DUFFY, 2011, p.9). Esta faceta vinculante se compagina con una dimensión "indirecta" del cuidado, comprendida como: 
La transferencia que hace algún sistema social -especializado o no-delosmecanismosnecesariosparaquelosindividuosgeneren por cuenta propia las atenciones que requieren. Básicamente, el cuidado indirecto se materializa en la transferencia de recursos: pensiones de vejez, apoyo económico de familiares, limosnas u otras prestaciones asistenciales (GIMÉNEZ, 2004, p.107).

Diversos autores alertan sobre la necesidad de superar perspectivas dicotómicas en este campo de estudios. Desde la globalización se ha observado que los cuidados adquirieron un carácter transnacional, desbordando bipolaridades conceptuales geográficas (distancia/proximidad). Pero desbordaron también esferas de acción (publico/privado), relacionales (biología/elección), morales (interés/altruismo), físicas (dependencia/autonomía) y temporales (tiempo de vida/tiempo de trabajo) (GONZÁLVEZ y ACOSTA, 2015, p.128).

Intentando comprender cómo todos estos elementos se combinan en acciones concretas, autoras feministas propusieron desglosar los "trabajos de cuidado" en tres tipos de actividades de carácter práctico (WAERNESS, 1987, pp.134-135). El cuidado directo dirigido a las personas: incluye el cuidado físico (alimento, baño, aseo), el emocional (escuchar, hablar, ofrecer consuelo) y los servicios (comprar comida, sacar de paseo). El mantenimiento físico es el cuidado de los espacios donde la persona vive (cambiar la ropa de cama, lavar la ropa, limpiar la habitación). Y el trabajo de parentesco: el fomento de relaciones y conexiones sociales entre el adulto mayor -él o ella- y las personas de su entorno, con las que tenga o no vínculos de parentesco sanguíneo (GLENN, 2010, p.5).

En las ciencias sociales, encontramos cinco abordajes teóricos que analizan los límites y posibilidades de estos trabajos de cuidado (ENGLAND, 2005, p.381): i) La perspectiva de la devaluación. Enfatiza que la baja recompensa salarial y de apoyo público que reciben los prestadores de cuidado deviene de discriminaciones de género, raciales y xenofóbicas: es indisociable de la feminización de estas labores y su asunción por mujeres de color y migrantes; 
ii) Marco del bien público. Asume que el trabajo de cuidados genera beneficios multidimensionales: ayuda el receptor a desarrollar habilidades, valores y hábitos que lo benefician a él y a otros; iii) Enfoque del prisionero del amor. Destaca que la baja remuneración del trabajo de cuidado se relaciona al aprovechamiento de la emocionalidad asociada al mismo: los empleadores sacan ventaja de las motivaciones altruistas de quienes cuidan y de los vínculos emocionales -empatía y apego- que ellos generan; iv) $L a$ mercantilización de la emoción. Argumenta que el capitalismo fuerza a los trabajadores del cuidado a actuar emociones (debido a la naturaleza esperada de sus labores), o a comercializar sentimientos profundamente íntimos (derivando en daños emocionales); v) El marco amor y dinero. Argumenta que la relación emociónremuneración no es dicotómica y que el trabajo de cuidados no es, necesariamente, más enajenante que otros.

No obstante, hay un componente de desigualdad de género que es transversal a estos debates. La reproducción de esta desigualdad está, ahora mismo, en tela de juicio debido a la inminente crisis de los cuidados que se está engendrando en contextos de transición demográfica acelerada, como el caso chileno. Con "crisis de los cuidados", nos referimos a la incapacidad -por parte de los Estados, sociedad civil y sujetos- de construir salidas articuladas para las necesidades presentadas por la población (PÉREZOROZCO, 2006, p.8). Los factores que configuran esta crisis pueden rastrearse a través de tres ejes que deben tomarse en cuenta para la construcción de políticas. Primero, las transformaciones sociodemográficas que, como venimos insistiendo, consolidan un incremento de la longevidad poblacional y de la demanda de cuidados. Segundo, los cambios sociolaborales que inciden en la disminución de mujeres que se ofrezcan para el oficio de prestar cuidados, debido al aumento del ingreso de mujeres al mercado laboral puertas afuera (EZQUERRA, 2012). Tercero, la adopción de directrices políticas neoliberales que, al incluir recortes sociales vigorosos, limitan la cobertura entre demanda y oferta de cuidados (PÉREZ-ORÓZCO, 2006, p.3). 


\section{Organización social de los cuidados y envejecimiento: familia, Estado, comunidad y mercado}

Terminamos el apartado anterior definiendo que la crisis de los cuidados alude a transformaciones sociodemográficas, sociolaborales y de orientación de las políticas económicas. Quedó pendiente mencionar que esta crisis también alude a la desestabilización del modelo tradicional de "organización social del cuidado". Esta última se refiere a la manera como cada sociedad establece una correlación entre sus necesidades de cuidados y la forma como les da respuesta. Es el modo como los actores sociales que pueden tener un papel en la provisión de cuidados -la familia, el Estado, la comunidad y el mercado- se combinan para esta provisión y también el protagonismo que asume cada uno de ellos (ARRIAGADA, 2010, p.59). La provisión de cuidados no ocurre de manera aislada o estancada: ella resulta de una continuidad en la que se suceden actividades, trabajos y responsabilidades asumidos por estos diferentes actores (RODRÍGUEZ, 2015, p.40). Así, es importante enfatizar que estas diversas esferas actúan de forma interrelacionada e interdependiente. Incluso cuando esta interpelación no resulta de una planificación o apuesta clara, lo cierto es que la acción en cada una de estas esferas interfiere en las demás. Y esta interrelación implica la existencia de contactos, continuidades y rupturas entre actores que transitan entre la familia, el Estado y el mercado (en papeles diversos y heterogéneos).

El incremento de la longevidad en países como Chile provoca el surgimiento de nuevas necesidades con relación a las pensiones y a la seguridad social (GIMÉNEZ 2004; MARCO, 2004; OECD, 2015), a la salud (MENÉNDEZ et al., 2005; SANHUEZA et al., 2005), a la vivienda y a la accesibilidad y uso de espacios públicos (MORENOS y CORREGIDOR, 2010). Estas necesidades se manifiestan de modo heterogéneo, pues el acceso a los recursos que posibilitan el cuidado son diferenciados de acuerdo al nivel socioeconómico, al género, la localización espacial y la edad de las 
personas (GONZÁLVEZ, 2017). La OIT (2009, pp.1-3) sintetiza este cuadro explicando que, en América Latina, existen cada vez menos personas disponibles dentro de las familias para hacerse cargo de las personas mayores; menos niños y jóvenes con quienes compartir las tareas de cuidado; insuficientes servicios de salud para los mayores; escaso acceso a los planes de pensiones y una exclusión de los mayores del mercado laboral formal. Es un hecho que la mayoría de los adultos mayores dependen de sus familias para satisfacer sus necesidades. La situación demanda plantearse seriamente cómo estas nuevas necesidades serán resultas y a quiénes les corresponderá hacerse cargo de ellas.

A continuación, analizamos los desafíos y posibilidades de la familia, del Estado, de la comunidad y del mercado en su interacción (en diversos grados y con distintos niveles de responsabilidad) en la cobertura de servicios directos e indirectos para satisfacer las necesidades de cuidado de los adultos mayores.

\section{Familia}

Es posible identificar cuatro apreciaciones bastante naturalizadas sobre la relación entre las familias y los cuidados. Primero, que los trabajos del cuidado debieran circunscribirse al "espacio privado", acotándose a la intimidad familiar (FILGUEIRA, 2009, p.112). Segundo, que las mujeres serían las responsables por ellos dentro y fuera de las familias (ARONSON, 1992, TREVIÑO et al., 2006, ARRIAGADA, 2009, GLENN, 2010). Tercero, la desconfiguración de las fronteras entre trabajos de cuidado, domésticos y reproductivos, asumidos frecuentemente como intercambiables entre sí (DUFFY, 2011, p.12). Cuarto, la centralización del afecto (entendido como práctica "femenina") como un elemento estructurante de los contenidos y motivaciones del cuidado, en oposición a los trabajos "más racionalizados" 
vinculados a la "esfera pública" (asociados a la masculinidad, productividad y mercado) (DUFFY, 2011, p.12).

Se sostiene, en diversos países latinoamericanos, la apreciación de que la familia -y dentro de ella, las mujeres- constituyen el "pilar" del cuidado. Esta idea naturaliza la división sexual del trabajo y los roles socioculturales atribuidos a las mujeres. Invisibiliza, además, el hecho de que los Estados reproduzcan y avalen, en su legislación y políticas públicas, esta distribución de los cuidados. Diversos investigadores observaron esta tendencia para el caso de Chile (ZARIT et al., 1998, p.499). Pero los cambios sociales y demográficos vividos por el país ponen estas naturalizaciones en entredicho, porque desconocen la drástica reorganización de los cuidados al interior de las familias. Estas transformaciones serían referentes: i) al tipo y duración de las uniones de pareja; ii) a las pautas reproductivas (sobre la decisión de embarazarse o no, de cuándo hacerlo y de cuántos hijos/as tener); iii) a la división sexual del trabajo; iv) a la combinación entre ciclo vital individual y familiar (FILGUEIRA, 2009, p.112).

Estos debates traen a luz elementos de reflexión obligada para el desarrollo de las políticas públicas de atención a la vejez en Chile. El primero de estos puntos se refiere a la sobrecarga femenina en el desempeño de los trabajos del cuidado. En el curso de vida de las mujeres, las labores del cuidado constituyen actividades no remuneradas y sin reconocimiento social (ARONSON, 1992; AGUIRRE, 2007; GLENN, 2010; DUFFY, 2011; COMAS, 2017), que las empujan hacia procesos de marginación y vulneración (OIT, 2009). Su sobrecarga tiende a magnificar interseccionalmente las desigualdades que ellas experimentan a niveles sociales, económicos, educacionales, laborales e incluso normativos, ensanchando así las brechas de género producidas en el curso de su vida productiva.

Las responsabilidades de provisión de cuidados se prolongan e intensifican en el envejecimiento: las mujeres mayores son presionadas a actuar como cuidadoras - de sus nietos/as, parejas e hijos/as, por ejemplo-, pese a necesitar, también ellas, de 
crecientes cuidados (GLENN, 2010, p.88). Muchas son presionadas, incluso, a actuar también como pilares de los cuidados comunitarios, compaginando frecuentemente estos ámbitos con sus actividades remuneradas (formales o informales) (ARONSON, 1992, p.25). Simultáneamente, es posible identificar otros elementos que potencian el desmedro de las mujeres mayores con relación a sus pares masculinos: las menores pensiones, la vulnerabilidad económica y la mayor dependencia hacia las instituciones estatales de asistencia social (KNODEL y OFSTEDAL, 2003; MONTAÑO, 2004; GIMÉNEZ, 2004; MARCO, 2004; CALASANTI et al., 2006). Si observamos la composición media de las fuentes de renta de personas con más de 60 años en Chile, tenemos que el $54,3 \%$ de los hombres cuenta con ingresos procedentes del trabajo, cifra que se reduce a solamente un $27,4 \%$ en el caso de las mujeres (CASEN, 2013, p.24). Entre las mujeres de más de 60 años, predominan los "subsidios monetarios" como principal fuente de ingreso, principalmente en el primer quintil (pensión básica solidaria y aporte solidario). Así, las mujeres que acuden al sistema solidario de protección social lo hacen porque el monto que resulta de su cotización es escaso o inexistente, impidiéndoles cubrir integralmente las necesidades propias de la vida cotidiana (alimentación, vestuario, salud y vivienda).

Con todo, las contradicciones del proceso demandan una mirada analítica atenta a los matices. Las mujeres mayores también cuentan con "herramientas" socio-relacionales de género que las disponen más favorablemente al enfrentamiento de las problemáticas del envejecimiento. Como mencionamos páginas atrás, la realización de actividades consideradas "tradicionalmente femeninas", como la creación de lazos sociales, les permite sortear de manera más llevadera algunas de las necesidades que caracterizan la vejez (KNODEL y OFSTEDAL, 2003, pp.680-681).

Asimismo, la obligación moral de cuidar de los padres cuando estos envejecen recae, mayoritariamente, sobre las hijas (POST, 1990, p.85). Desde los años ochenta, las investigaciones sociológicas arrojan evidencia de que las mujeres desean que sus 
hermanos varones compartan igualitariamente el cuidado de los padres mayores. Estos estudios demuestran, además, que los hijos prefieren comprometerse con formas indirectas de cuidado (compromisos financieros o arreglos a distancia), evitando el cuidado directo. Generalmente, son las mujeres quienes están "asignadas" al rol de cuidadoras directas, sin que su consentimiento o sus necesidades sean "seriamente considerados" (POST, 1990, p.85). Pero no se trata solamente de que no todos en las familias se hagan cargo del cuidado directo. Estudios efectuados en Chile -como el de FLORES et al. (2012, p.29) sobre cuidados personas en situación de dependencia- encontraron que las necesidades de cuidado de las mujeres cuidadoras son, por lo general, desatendidas. El Servicio Nacional del Adulto Mayor, senama, lo confirma, indicando que las mujeres cuidan al menos doce horas diarias, no comparten el cuidado y se sienten solas, sobrecargadas y sobrepasadas por su situación. Agrega que, en Chile, dos tercios de las mujeres cuidadoras no ha tomado vacaciones hace más de cinco años (SENAMA, 2010). Esto genera preocupaciones respecto de elementos sociales, económicos y de salud que pueden afectar a las mujeres cuidadoras. Entre ellos, el denominado "Síndrome del Cuidador": un proceso de enfermedad por sobrecarga física y emocional verificado entre mujeres que se hacen cargo de familiares que demandan cuidados intensivos -adultos mayores 0 personas con discapacidad- (FLORES et al., 2012, pp.31-32).

En suma: la familia sigue siendo el punto neurálgico de organización del cuidado social en Chile y esta realidad reproduce la inequidad de género, proyectando un ensanchamiento de las brechas de derechos a los que acceden las mujeres en la vejez.

\section{Estado}

Entre 1980 y 1990, las reformas neoliberales en América Latina impulsaron la privatización (parcial o completa) de los sistemas 
Envejecimiento, género y cuidados: debates para situar las políticas públicas Herminia Gonzálvez Torralbo • Sofia Larrazabal • Menara Guizardi

de pensiones y demás estructuras de amparo al envejecimiento (sistemas sanitarios, recreativos, psicosociales). Pero pese al impacto y a la envergadura de estas reformas, los Estados de la región siguen teniendo un papel fundamental en la entrega de servicios asistenciales y en la administración y provisión de mecanismos compensatorios para los ciudadanos de la tercera edad: especialmente a los que no logran acceder a pensiones contributivas (MARCO, 2004, p.32).

Sería imposible analizar aquí las particularidades del fenómeno en diferentes contextos latinoamericanos. Acorde a nuestros objetivos, nos limitaremos al papel que viene desempeñando el Estado chileno con relación a la protección social de la vejez. La crisis demográfica enfrentada por el país tiene un impacto directo en el aumento de la presión social hacia una mayor intervención estatal en estas materias. Como las familias ya no cuentan con personas que se hagan cargo de la totalidad de las actividades de cuidado de los mayores (ZARIT et al., 1998, pp.499-500), deben externalizar estas necesidades hacia el sector público (o hacia el privado, como abordaremos a continuación).

Desde 2012, el Estado chileno viene siendo fuertemente presionando por la sociedad civil a asumir su rol constitucional de garante de la atención a las necesidades integrales de los ciudadanos (especialmente de los más vulnerables), garantizando el acceso público a los servicios sociales y de salud (HUENCHUÁN, 2014, p.159). Esta demanda se traduce en requerimientos al Estado de hacerse cargo -a través de sus divisiones y subdivisiones ministeriales- de la entrega (directa e indirecta) de servicios que permitan la subsistencia de los mayores en condiciones de vida aceptables. En este contexto se enmarca la creación de una Política Integral de Envejecimiento Positivo para Chile, pensada como un plan estratégico para el periodo 2012-20254.

\footnotetext{
4 Las primeras aproximaciones estatales al escenario del envejecimiento se sitúan en 1995 con la creación de la Comisión Nacional para el Adulto Mayor, órgano asesor de la Presidencia. Posteriormente, en 1996 se aprueba la Política Nacional para el Adulto Mayor, creándose en 1997 los Comités Regionales para el Adulto Mayor. En 1999, durante el año internacional del adulto mayor, se envió al Congreso el proyecto de ley para crear el Servicio Nacional del Adulto Mayor (SENAMA), aprobado en 2002. Pasados 10 años de funcionamiento de esta institución, se crea la Política Integral de Envejecimiento Positivo para Chile 2012-2025 (Ganga et al. 2016, p.183).
} 
Los diagnósticos sociales realizados para la construcción de este plan atestiguan que los dos mayores gastos del Estado con las personas mayores eran, en 2012, referentes a las pensiones (74\% del presupuesto del ítem) y a la salud (25\%) (MINDES, 2012, p.29). Pero hay una creciente preocupación estatal en Chile sobre la necesidad de avanzar hacia políticas del cuidado activo de la población en edad mayor. En gran medida, esta preocupación responde a la entrada del país en la Organización para la Cooperación y el Desarrollo Económicos (OCDE), proceso que catalizó la percepción de diversos procesos sociales que, hasta el momento, habían recibido atención insuficiente por parte del Estado. Este giro aparece con alguna transparencia en los documentos del Ministerio de Salud (MINSAL) y del Ministerio de Planificación (MIDEPLAN), en los cuales se empieza a abordar la necesidad de pensar los "cuidados a largo plazo" como una responsabilidad estatal (SUPER SALUD et al., 2008, p.2).

La forma de implementar el cuidado a través del Estado fue influida por la perspectiva del "envejecimiento activo" o "positivo", que vertebra la Política Integral de Envejecimiento Positivo, planteando que la vejez debe ser entendida desde sus potencialidades y no solamente como un desmedro de capacidades. Con todo, el discurso de varios ministerios implicados en la cuestión del envejecimiento asume que la resolución de problemas, necesidades y también la promoción de autonomía y autovalencia de la población mayor es una forma de reducir los gastos estatales futuros por parte de los sistemas de salud (SUPER SALUD/MINSAL/MIDEPLAN, 2008, 13). Prima, así, una lectura de reducción de daños que -pese a garantizar un avance en la cobertura de los derechos de la población mayor-se plantea desde lineamientos utilitaristas, reconociendo, pero sin afrontar, las desigualdades producidas por la reducción de la infraestructura estatal en áreas de atención social y sanitaria (como resultado de décadas de reformas neoliberales).

La Organización Mundial de la Salud (OMS, 2016) indica que las políticas estatales sobre la vejez y en materia de cuidados 
debieran contemplar prestaciones vinculadas a servicios de seguridad social (de diversa naturaleza y enfoque), además de la salud. En Chile, la entidad estatal encargada de velar por la plena integración de las personas mayores a la sociedad, su protección ante el abandono e indigencia, y el ejercicio de los derechos que el marco legal nacional reconoce es el Servicio Nacional del Adulto Mayor (SENAMA) (GANGA et al., 2016, p.183).

EI SENAMA (2009) promueve la articulación de seis tipos de acciones o servicios de cuidado para las y los adultos mayores: i) Programas para la promoción de salud y el autocuidado; ii) Cuidados y servicios médicos ambulatorios; iii) Centros abiertos diurnos; iv) Atención de cuidados paliativos domiciliarios; v) Cuidados residenciales temporales y; vi) Cuidados residenciales permanentes. Pese al papel protagónico del SENAMA, estas acciones demandan un abordaje intersectorial, con la participación de otras divisiones ministeriales, dependientes de los ministerios de Salud, de Desarrollo Social, de Economía, Fomento y Turismo, de Educación, de Vivienda y Urbanismo y del Ministerio Secretaría General de Gobierno (GANGA et al., 2016).

\section{Comunidad}

Cuando hablamos de "cuidados comunitarios" aludimos a una serie de actividades que, muy a menudo, interconectan la participación de estructuras estatales, de las familias y del mercado. El ámbito comunitario constituye el espacio de entrecruce de diversos actores que intervienen en los cuidados, pero esto no significa que la comunidad esté subsumida por estos actores o que equivalga a ellos. El espacio comunitario es, en este sentido, un ámbito sui generis. En él, los cuidados pueden involucrar servicios provistos en residencias con locaciones, a través de la colocación de diversos tipos de empleados públicos en la comunidad; gestionados por los propios residentes de manera voluntaria o por amigos, 
vecinos, y miembros de aquellas asociaciones (profesionales, sindicales, culturales, religiosas) de las cuales participan algunos 0 todos los miembros de la comunidad (WAERNESS, 1987, p.133). Así, independientemente de la forma como el cuidado comunitario sea provisto, su diferencial se refiere a que actúa, moviliza, articula y refuerza las redes sociales -tanto las directas como las indirectas-, ofreciendo una vinculación de las personas mayores con el entorno. Ya sean gratuitos o pagados, los servicios, ayudas o prestaciones de estos cuidados mantendrán siempre una operabilidad en red. De ahí su adjetivación "comunitaria".

A lo largo del territorio chileno, observamos las asociaciones, clubes y grupos organizados por las comunidades que gestionan, por ejemplo, la recreación y las actividades fuera de casa para los adultos mayores. Algunos estudios han verificado, además, la importancia de las enfermerías comunitarias, las cuales cumplen un rol de apoyo social, paliando la sobrecarga del cuidador familiar (FLORES et al., 2012, p.33). Este alcance ratifica que los cuidados comunitarios tienen su valoración no solo por la cobertura de necesidades de los mayores, sino porque significa una disminución de la sobrecarga femenina.

En las Encuestas de Calidad de Vida en la Vejez realizadas en Chile en 2010 y 2013 (SENAMA y PUC, 2011, 2014) se comprenden las actividades sociales de carácter comunitario como factores que promueven un envejecimiento "activo y saludable". Según la encuesta de $2013,49 \%$ de los adultos mayores afirma que las actividades en que más participan son grupos religiosos, clubes de adultos mayores, organizaciones de barrio o juntas de vecinos. El diagnóstico arroja, además, que las mujeres mayores salen menos de sus casas -se socializan menos en el espacio público-, pero están más involucradas en actividades colectivas, debido, precisamente, a su activa afiliación a estos grupos religiosos, barriales, culturales. Otros diagnósticos sociales coinciden en que los adultos mayores que participan de estas redes u organizaciones comunitarias tienden a encontrar en ellas soluciones para muchas de sus problemáticas cotidianas (SENAMA, 2009, p.23) y se sienten 
más a gusto o más satisfechos que los demás con la experiencia del envejecimiento (SENAMA y PUC, 2014).

En varias regiones chilenas las municipalidades forman parte de esta red de actividades comunitarias para la tercera edad a través de la organización de los clubes y talleres de personas mayores. Estos abarcan una serie de necesidades, incluyendo aquellas relacionadas con la mantención física y de la salud (con actividades de gimnasia o la consulta médica a especialistas de la salud dentro de los centros abiertos de atención diurna) y a la asistencia psicológica, mental y psicosocial. Se observa, no obstante, una enorme disparidad entre los recursos que las municipalidades disponen para este fin. Lo anterior puede ser motivado tanto por los distintos grados de importancia que se da políticamente al tema del envejecimiento, como por los desiguales niveles de ingresos fiscales y de recursos generales con que cuentan los municipios chilenos para actividades de corte social. Asimismo, el SENAMA (2009) indica que muchos de estos talleres y clubes municipalizados cuentan con recursos facilitados por el Estado (y vinculados a presupuestos ministeriales y de subsecretarías nacionales, como la Subsecretaría de Desarrollo Regional y Administrativo y la Subsecrería de Servicios Sociales).

No solamente las municipalidades y el Estado vierten recursos, infraestructuras y servicios en estas actividades de carácter comunitario, en todo el territorio nacional se observa también la presencia de fundaciones de beneficencia como el Hogar de Cristo, la Fundación Las Rosas o la Sociedad San Vicente de Paul. Algunas instituciones eclesiásticas cuentan también con residencias de larga estadía y programas específicos de atención para adultos mayores (que, por lo general, se profesan como instituciones sin fines de lucro). Su papel social es considerable, puesto que atienden precisamente a aquellos adultos mayores que no acceden a una pensión o que tienen solamente una pensión asistencial. Las personas suelen ser derivadas desde distintos programas de gobierno, lo que no deja de implicar una tercerización, por parte del Estado, de su responsabilidad constitucional de la atención 
y cuidado de las poblaciones en condición de vulneración. Nada de lo dicho hasta aquí se refiere al sector más vulnerable, que no aparece reflejado en las políticas públicas y solo recibe atención paliativa por parte de agrupaciones de particulares solidarios: las personas -NNA, adultos y ancianos de ambos sexos- en situación de calle.

\section{Mercado}

Es posible decir que, en Chile, el sector privado viene funcionando como un punto de bisagra entre el Estado y la familia. Como decíamos páginas atrás, esto se debe, parcialmente, a la pérdida de capacidad (en tiempo y recursos) de las familias para cuidar de los mayores. En la ausencia de una red pública que dé respuestas a las necesidades de esta población, el mercado se presenta como una solución -cada vez más a menudo, como la única solución- disponible.

Por su parte, el propio Estado chileno tiende a tercerizar y externalizar varias de las prestaciones de cuidado, involucrando en esto tanto las empresas privadas, como las fundaciones, religiosas - laicas. Esta modalidad no deja de constituir un proceso de estatización del mercado, en la medida en que son los subsidios públicos los que son entregados a las empresas de cuidado para que se hagan cargo de la población mayor. Esta situación se viene complejizando exponencialmente con el sostenimiento del proceso de envejecimiento de la población chilena.

El acceso a estas prestaciones privadas en Chile es escalonado de acuerdo con el ingreso familiar y con la capacidad adquisitiva individual de las personas mayores. El mercado ofrece establecimientos de larga estadía, centros de día y atención primaria, programas de cuidado domiciliarios y programas de cuidado preventivo, los cuales pueden variar en calidad, cantidad 
y precio. Este último puede oscilar en hasta en un mil por ciento, dependiendo de las características y prestaciones ofrecidas.

El Ministerio de Salud regula las disposiciones técnicas que el sector privado debe atender para poder mantener estos establecimientos. Por ejemplo, las residencias de larga estadía deben contar con enfermeras/os, médicas/os y nutricionistas. En esta clasificación se pueden encontrar residencias que ofrecen servicios mínimos, hasta establecimientos de lujo, catalogados como los "senior homes" encontrados en las comunas de niveles socioeconómicos más altos (en la Región Metropolitana, ellas están en Las Condes, Providencia, Vitacura) (SENAMA, 2009). Un segundo tipo de residencias de larga estadía son los "hogares", las cuales constituyen una alternativa para mayores con menor poder adquisitivo. Las fiscalizaciones sanitarias han encontrado, en los últimos años, hogares de este tipo con condiciones mínimas de comodidad, con un equipamiento inferior en calidad y cantidad y personal de trato directo sin calificación.

La heterogeneidad de los servicios y la lógica propia del mercado para proveerlos -adscripta a principios de lucro y/o rentabilidad económica- son elementos que demandan una activa participación reguladora por parte del Estado, aspecto central para pensar la articulación de las políticas sociales sobre la vejez en Chile.

\section{Consideraciones finales}

Quisiéramos sintetizar aquí algunas de las observaciones que nuestra revisión permite elaborar sobre el cruce entre el proceso de envejecimiento, su feminización y las demandas de cuidado social de las poblaciones mayores.

Un primer punto, en este sentido, refiere a la constatación de que el desajuste entre necesidades, recursos y ofertas reales de cuidados hacia la población envejecida interpela de manera 
particular a las mujeres mayores. En el panorama demográfico de envejecimiento que enfrenta Chile, las mujeres mayores son crecientemente interpeladas por el dilema de los cuidados: cuando no se hacen cargo directamente de ellos, se les imputa la responsabilidad de decidir quién o quiénes deberían hacerse cargo de proveerlos.

Este proceso está influido, como hemos discutido, por la naturalización de la vinculación entre los cuidados y las familias, la cual reproduce desigualdades estructurales de género. Se naturaliza que las mujeres son quienes deben cuidar dentro de las familias y esta sobrecarga femenina tiene un carácter duradero, que atraviesa el todo el ciclo vital de las mujeres, en ocasiones desde la niñez, magnificándose con el envejecimiento. En parte porque las mujeres pasan, en esta etapa, a demandar ellas mismas más cuidados. Y en parte debido a la condensación, en la vejez, de las desigualdades sociales, económicas, educacionales y laborales que ellas van acumulando a lo largo de su ciclo vital.

La suma de estos aspectos indica que las mujeres mayores se ven presionadas con relación a los cuidados, debiendo balancear roles individuales y colectivos. Ellas equilibran el autocuidado, con el cuidado hacia otros, usualmente miembros de la familia, aunque sin descartar que cuiden también a otros miembros de la comunidad. Y compaginan todo esto, frecuentemente, con el desempeño de trabajos remunerados. Asimismo, son las mujeres las principales agentes de las iniciativas colectivas de soporte de la vejez comunitaria. Por tanto, es posible concluir que las prácticas de cuidar y ser cuidado afectan a los significados asociados a la vejez, pero de forma particularmente feminizada.

Considerando todos estos aspectos, una política pública que planteara la equidad de cuidados de la vejez debiera operar en favor de subsanar las asimetrías que la sobrecarga de género produce. Es decir, dicha política debe estructurarse desde una mirada transversal e intergeneracional de género. Quisiéramos apuntar seis directrices en este sentido. 
Primero, estas políticas deben partir por la adopción de un principio de justa igualdad de oportunidades sobre la división del cuidado dentro de las familias, potenciando igualitariamente las capacidades, entre mujeres y hombres, para competir por posiciones de poder y autoridad fuera de la familia. Segundo, deben plantear el establecimiento -a través de una legislación pertinente-, de límites para el deber del cuidado, fundados en la libertad moral de aceptar su ejercicio o rechazarlo. Tercero, deben establecerse mecanismos de concienciación social que planteen que la asistencia hacia las generaciones mayores es responsabilidad de todos y todas (JECKER, 2002, pp.129-130).

En cuarto lugar, es fundamental que la política pública logre superar las limitaciones de una visión de los cuidados circunscritos a los afectos, precisamente porque este encapsulamiento refuerza los dispositivos simbólicos que justifican o preconfiguran la sobrecarga femenina con relación a ellos (como discutimos en el segundo apartado). Desde nuestra perspectiva, la política pública debe plantearse el problema de los cuidados de la vejez a través de una mirada interseccional: que contemple la interconexión de las desigualdades clase y género; pero enfatizando seriamente los problemas de igualdad engendrados por la división del trabajo de cuidado a través de líneas étnicas y/o raciales. El desafío de esta perspectiva de la política pública es, precisamente, hacer confluir las acciones estatales existentes que preconizan la igualdad distributiva de las responsabilidades de los cuidados y de otras formas de desigualdad socialmente establecidas.

Debido a lo anterior y, en quinto lugar, estas políticas debieran plantearse intersectorialmente: conectando crecientemente a diferentes subdivisiones del Estado (ministerios, secretarías, oficinas, servicios). Es más, como también han reflexionado FLORES et al. (2012, p.31), debido a las particularidades de las necesidades de las personas mayores (especialmente aquellas en cuadro de dependencia), las políticas públicas sobre la vejez difícilmente podrían ejecutarse sin que el Estado asuma un rol de articulador de las estructurales sociales de cuidado desarrolladas desde las 
familias, comunidades y mercado. En suma: la maximización de los resultados de las intervenciones de las políticas estatales demanda articular el Estado y los demás actores sociales proveedores del cuidado (AGUIRRE, 2007, p.6).

Finalmente, nuestro último punto de reflexión se vincula a la necesidad de que estas políticas públicas también partan de una escucha comprometida de las visiones de mundo, necesidades y propuestas de las personas mayores. La dimensión participativa de estas políticas es imposible si no nos planteamos escuchar lo que las personas mayores tienen para decirnos sobre los significados de la vejez, antes de que podamos responder a sus necesidades y a las nuestras de manera relevante y efectiva.

\section{Referencias}

AGAR, Lorenzo. Envejecimiento en América Latina y el Caribe: hechos sociodemográficos y reflexiones éticas. Acta Bioethica, Santiago, v. 7, n. 1, 2001, pp. 27-41.

AGUIRRE, Rosario. Familias como proveedoras de servicios de cuidados. In: ASTELARRA, Judith (coord.). Género y cohesión social. Madrid: Fundación Carolina, 2007, pp. 83-94.

ARANCO, Natalia, STAMPINI, Marco, IBARRARÁN, Pablo y MEDELLÍN, Nadin (eds.). Panorama de envejecimiento y dependencia en América Latina y el Caribe. Washington: BID, 2018. ARONSON, Jane. Women's Sense of Responsibility For The Care of Old People: 'But Who Else Is Going to Do It?' Gender \& Society, Chicago, v. 6, n. 1, Mar. 1992, pp. 8-29.

ARRIAGADA, Irma. La crisis de cuidado en Chile. Revista de Ciencias Sociales, Montevideo, v. 23, n. 27, 2010, pp. 58-57. BIBLIOTECA NACIONAL DE CHILE [BCN]. El futuro demográfico de América Latina. Programa Américas, 2016. Net, Santiago, 2016. 
Disponível em: https://www.bcn.cl/observatorio/americas/noticias/el-futuro-demografico-de-america-latina Acceso em: 9 julio 2018.

BUTLER, Robert. Age-ism: Another form of bigotry. The Gerontologist, Oxford, v. 9, n. 4, 1969, pp. 243-246.

CALASANTI, Tony, SLEVIN, Kathleen y KING, Neal. Ageism and Feminism: From "Et Cetera" to Center. NWSA Journal, Baltimore, v. 18, n. 1, 2006, pp. 13-30.

CASEN. Adultos Mayores. Síntesis de Resultados. Documento de trabajo, 2013. Net, Santiago, marzo 2013. Disponível em: http:// observatorio.ministeriodesarrollosocial.gob.cl/documentos/ Casen2013_Adultos_mayores_13mar15_publicacion.pdf. Acceso em: 9 julio 2018.

CELADE. Proyecciones de población. Santiago: CEPAL-CELADE, 2014.

CEPAL. La transición demográfica en América Latina. Santiago: CEPAL, 2000.

CHAKIEL, Juan. El envejecimiento de la población latinoamericana: ¿Una relación de dependencia favorable? Santiago: CEPAL, 2000.

COLEMAN, Lisa y WATSON, Sophie. Women over Sixty. A study of the Housing, Economic and Social Circumstances of Older Women. Canberra: Australian Institute of Urban Studies, 1987.

COMAS, Dolors. El don y la reciprocidad tienen género: las bases morales de los cuidados. Quaderns-e, Barcelona, v. 22, n. 2, 2017, pp. 17-32.

DATAN, Nancy. Aging women: the silent majority. Women's Studies Quarterly, New York, v. 17, n. 1/2, 1989, pp. 12-19.

DEL VALLE, Alejandro. Bienestar, familia y problemas de cuidados en América Latina. Emancipação, Ponta Grossa, v. 13, n. 3, 2014, pp. 27-45. 
DONOSO, Enrique, CARVAJAL, Jorge y DOMÍNGUEZ, María Angélica. Reducción de la fecundidad y envejecimiento de la población de mujeres chilenas en edad fértil: 1990-2004. Revista Médica de Chile, Santiago, n. 137, 2009, pp. 766-773.

DUFFY, Mignon. Making care count. A century of gender, race and paid care work. New Jersey: Rutgers University Press, 2011.

ENGLAND, Paula. Emerging theories of care work. Annual Review of Sociology, Chicago, n. 31, 2005, pp. 381-399.

ESTES, Caroll. The aging enterprise. San Francisco: Jossey-Bass, 1979.

ESTES, Caroll. The aging enterprise revisited. The Gerontologist, Oxford, v. 33, n. 3, pp. 1993, 291-298.

ESTES, Caroll, GERARD, Lenore y CLARKE, Adele. Women and the economics of aging. International Journal of Health Services: Planning, Administration, Evaluation, Westport, v. 14, n. 1, 1984, pp. 55-68.

EZQUERRA, Sandra. Sobre viejas y nuevas gestiones de la crisis o el retorno de las mujeres al hogar. Viento Sur, Madrid, n. 121, 2012, pp. 87-95.

FERNÁNDEZ, José Manuel y KEHL, Susana. La construcción social de la vejez. Cuadernos de Trabajo Social, Madrid, 2001, v. 14, pp. 125-161.

FILGUEIRA, Fernando. El desarrollo maniatado en América Latina. Buenos Aires: CLACSO, 2009.

FLORES, Elizabeth, RIVAS, Edith y SEGUEL, Fredy. Nivel de sobrecarga en el desempeño del rol del cuidador familiar de adulto mayor con dependencia severa. Ciencia y Enfermería, Concepción, v. 28, n. 1, 2012, pp. 29-41.

GANGA, Francisco, PIÑONES, María Angélica, GONZÁLEZ, Diego y REBAGIALTI, Francisca. Rol del Estado frente al envejecimiento de 
la población: el caso de Chile. Convergencia, Ciudad de México, v. 23, n. 71, 2016, pp. 175-200.

GIBSON, Diane. Broken Down by Age and Gender: The Problem of Old Women Redefined. Gender \& Society, Chicago, v. 10, n. 4, 1996, pp. 433-448.

GIMÉNEZ, Daniel. Género, previsión y ciudadanía social en América Latina. In: MARCO, Flavia (coord.). Los sistemas de pensiones en América Latina: un análisis de género. Santiago: CEPAL, 2004, pp. 99-152.

GLENN, Evelyn. Forced to care: Coercion and caregiving in America. Cambridge, MA: Harvard University Press, 2010. GONZÁLVEZ, Herminia. Ser Mujer Mayor en Santiago de Chile: feminización de los cuidados en la vejez y desigualdades acumuladas. In: VERA, Antonieta (ed.). Malestar social y desigualdades en Chile. Santiago, Ediciones UAH, 2017, pp. 173-195.

GONZÁLVEZ, Herminia; ACOSTA, Elaine. Cruzar las fronteras desde los cuidados: la migración transnacional más allá de las dicotomías analíticas. In GUIZARDI, Menara (ed.). Las fronteras del Transnacionalismo. Límites y desbordes de la experiencia migrante en el centro y norte de Chile. Santiago, Ocho Libros, 2015, pp. 126-150.

HESS, Beth. Aging policies and old women: The hidden agenda. In: ROSSI, Alice (ed.). Gender and the life course. Nueva York, NY: Polity, 1985, pp. 319-322.

HUENCHUÁN, Sandra. “QQué más puedo esperar a mi edad?". Cuidado, derechos de las personas mayores y obligaciones del Estado. In: HUENCHUÁN, Sandra y RODRÍGUEZ, Rosa (eds.). Autonomía y dignidad en la vejez: teoría y práctica en políticas de derechos de las personas mayores. Ciudad de México: CEPAL, 2014, pp. 153-168.

HUTHEESING, Otome. Facework of a female elder in a Lisu field, Thailand. In: BELL, Diane, CAPLAN, Patricia y WAZIR-JAHAN 
BEGUM Karim (eds.). Gendered fields. Women, men and ethnography. Londres: Routlegde, 1993, pp. 93-102.

INSTITUTO NACIONAL DE ESTADÍSTICAS DE CHILE [INE]. Resultado de población por sexo y edad. Santiago: INE, 2017. IZQUIERDO, María Jesús. Del sexismo y la mercantilización del cuidado a su socialización: hacia una política democrática del cuidado. Barcelona: Emakunde, 2003

JECKER, Nancy. Taking care of one's own: justice and family caregiving. Theoretical Medicine \& Bioethics, Amsterdam, v. 23, n. 2, 2002, pp. 117-133.

KNODEL, John y OFSTEDAL, Mary Beth. Gender and Aging in the Developing World: Where are the men? Population and Development Review, New York, NY, v. 29, n. 4, 2003, pp. 677-698.

LAMAS, Marta. Usos, dificultades y posibilidades de la categoría género. Papeles de Población, Toluca, v. 5, n. 21, 1999, pp. 10-61. LEVY, Judith. Intersections of gender and aging. The Sociological Quarterly, Ames, v. 29, n. 4, 1988, pp. 479-486.

MARCO, Flavia. Rasgos generales de los sistemas previsionales de capitalización individual y de sus contextos laborales y demográficos. In: MARCO, Flavia (coord.). Los sistemas de pensiones en América Latina: un análisis de género. Santiago: CEPAL, 2004, pp. 31-58.

MARSHALL, Leni. Aging: A feminist issue. NWSA Journal, Baltimore, v. 18, n. 1, pp. 7-13, 2006.

MENÉNDEZ, Jesús, GUEVARA, Adialys, ARCIA, Néstor, LEÓN, Esther María, MARÍN, Clara y ALFONSO, Juan. Enfermedades crónicas y limitación funcional en adultos mayores: estudio comparativo en siete ciudades de América Latina. Revista Panamericana de Salud Pública, Washington, DC, v. 17, n. 5/6, 2005, pp. 353-361. 
MINISTERIO DE DESARROLLO SOCIAL DE CHILE [MINDES].

\section{Política Integral de Envejecimiento Positivo para Chile 2012-}

2025. Santiago: Ministerio de Desarrollo Social, 2012.

MONTAÑO, Sonia. La agenda feminista y las reformas de los sistemas de pensiones en América Latina. In: MARCO, Flavia (coord.). Los sistemas de pensiones en América Latina: un análisis de género. Santiago: CEPAL, 2004, pp. 11-30.

MORENOS, David y CORREGIDOR, Ana Isabel. Urbanismo, espacio público y personas mayores: hacia la amabilidad de las formas.

Revista de Terapia Ocupacional de Galicia, A Coruña, n. 3, 2010, pp. 232-254.

NAZAR, Gabriela y FIGUEROA, Carolina. Creencias estereotípicas sobre el desempeño laboral de trabajadores mayores en Chile. Psicoperspectivas, Valparaíso, v. 14, n. 1, 2015, pp. 114-125.

OECD. Pensions at a glance 2015: OECD and G20 indicators. 2015. Net, Washington, octubre 2015. Disponível em: https://www. aranagenzia.it/attachments/article/7043/Pensions\%20at\%20a\%20 glance.pdf. Acceso em: 9 julho 2018.

OMS. World Health Statistics 2016: Monitoring Health for the SDGs. 2016. Net, Washington, DC, abril 2016. Disponível em: http://www.who.int/gho/publications/world_health_statistics/2016/en/ Acceso em: 3 mar. 2018.

OIT. Envejecimiento de la población: ¿quién se encarga del cuidado? Net, Washington, DC, maio, 2009. Disponível em: https:// www.oitcinterfor.org/sites/default/files/file_publicacion/nota_ trabfam8.pdf Acceso en: 3 mar. 2018

OSORIO, Paulina. La longevidad: más allá de la biología. Aspectos socioculturales. Papeles del CEIC, Bilbao, n. 22, 2006, pp. 1-28.

PEACE, Sheila. The forgotten female: Social policy and older women. In: PHILLIPSON, Chris y WALKER, Alan (eds.). Ageing and Social Policy: a Critical Assessment. Hants: Aldershot, 1986, pp. 61-86. 
PÉREZ ORÓZCO, Amaia. Amenaza tormenta: la crisis de los cuidados y la reorganización del sistema económico. Revista de Economía Crítica, Barcelona, 2006, n. 5, pp. 7-37.

PNUD. Desiguales. Orígenes, cambios y desafíos de la brecha social en Chile. 2017. Net, Santiago, junho 2017. Disponível em: http://www.cl.undp.org/content/chile/es/home/library/poverty/ desiguales--origenes--cambios-y-desafios-de-la-brecha-social-en-. html. Acceso en: 9 julho 2018.

POST, Stephen. Women and elderly parents: moral controversy in an aging society. Hypatia, Morelos, 1990, v. 5, n. 1, pp. 83-89.

RAMOS-TORO, Mónica. Mujeres mayores: Estudio sobre sus necesidades, contribuciones al desarrollo y participación social. Madrid, 2015. 488f. Tesis (Doctorado en Antropología Social) Universidad Autónoma de Madrid, Madrid, 2015.

RODRÍGUEZ, Corina. Economía feminista y economía del cuidado. Aportes conceptuales para el estudio de la desigualdad. Nueva Sociedad, Buenos Aires, n. 256, 2015, pp. 30-44.

RODRÍGUEZ, Juan, RUSSO, Moisés y CARRASCO, Marcela. Políticas públicas para una población que envejece: panorama y propuestas para el sistema de salud chileno. Temas de la Agenda Pública, v. 12, n. 92, 2017, pp. 1-13.

ROSENTHAL, Evelyn. Women and varieties of ageism. In: ROSENTHAL, Evelyn (ed.). Women, aging and and ageism. Londres: Harrington Park Press, 1990, pp. 1-6.

SANHUEZA, Marcela, CASTRO, Manuel y MERINO, José. Adultos mayores funcionales: un nuevo concepto en salud. Ciencia y enfermería, Concepción,. v. 11, n. 2, 2005, pp. 17-21.

SERVICIO NACIONAL DEL ADULTO MAYOR [SENAMA]. Estudio sobre la demanda de servicios de cuidado para las personas mayores. Santiago: SENAMA, 2009. 
SERVICIO NACIONAL DEL ADULTO MAYOR [SENAMA]. Estudio deja al Descubierto Importantes Diferencias Según Clase, Edad, Género y Zona de Residencia. Santiago: SENAMA, 2010.

SERVICIO NACIONAL DEL ADULTO MAYOR Y PONTIFICIA UNIVERSIDAD CATÓLICA DE CHILE. Chile y sus mayores: resultados segunda encuesta nacional calidad de vida en la vejez (2010). Santiago: PUC.

SERVICIO NACIONAL DEL ADULTO MAYOR Y PONTIFICIA UNIVERSIDAD CATÓLICA DE CHILE. Chile y sus mayores: resultados tercera encuesta nacional calidad de vida en la vejez (2013). Santiago: PUC.

SUPERINTENDENCIA DE SALUD [SUPER SALUD], MINSAL Y MIDEPLAN. Dependencia de los adultos mayores en Chile. Net, Santiago de Chile, agosto 2008. Disponível em: http://www.supersalud.gob.cl/documentacion/666/articles-4471_recurso_1.pdf. Acceso em: 3 mar. 2018.

TREVIÑO, Sandra, PELCASTRE, Blanca y MÁRQUEZ, Margarita. Experiencias de envejecimiento en el México rural. Salud Pública de México, Cuernavaca, v. 48, n. 1, 2006, pp. 30-38.

VELÁSQUEZ-GAVILANES, Raúl. Hacia una nueva definición del concepto "política pública". Desafíos, Bogotá, v. 20, 2010, pp. 149-187.

WAERNESS, Kari. A feminist perspective on the new ideology of community care for the elderly. Acta Sociólogica, Falun, v. 30, n. 2, 1987, pp. 133-150.

ZARIT, Steven, DAVEY, Adam, EDWARDS, Anne, FEMIA, Elia y JARROTT, Shannon. Family Caregiving: Research Findings and Clinical Implications. In: BELLACK, Allan y HERSEN, Michell (eds.). Comprehensive clinical psychology. Oxford: Elsevier, 1998, pp. 499-523. 